OPEN ACCESS

Edited by:

Luis Abel Quiñones,

University of Chile, Chile

Reviewed by:

Bruno Stieger,

University of Zurich, Switzerland

Domenico Criscuolo,

Genovax, Italy

*Correspondence:

M. Isabel Lucena

lucena@uma.es

Specialty section: This article was submitted to

Pharmacogenetics

and Pharmacogenomics,

a section of the journal

Frontiers in Pharmacology

Received: 15 July 2016 Accepted: 15 August 2016 Published: 26 August 2016

Citation:

Stephens C, Moreno-Casares A, López-Nevot M-Á, García-Cortés M, Medina-Cáliz I, Hallal H, Soriano G,

Roman E, Ruiz-Cabello $F$

Romero-Gomez M, Lucena Ml and

Andrade RJ (2016) Killer

Immunoglobulin-Like Receptor

Profiles Are not Associated with Risk of Amoxicillin-Clavulanate-Induced

Liver Injury in Spanish Patients.

Front. Pharmacol. 7:280

doi: 10.3389/fphar.2016.00280

\section{Killer Immunoglobulin-Like Receptor Profiles Are not Associated with Risk of Amoxicillin-Clavulanate-Induced Liver Injury in Spanish Patients}

\author{
Camilla Stephens ${ }^{1}$, Antonia Moreno-Casares², Miguel-Ángel López-Nevot ${ }^{2}$, \\ Miren García-Cortés ${ }^{1}$, Inmaculada Medina-Cáliz'1, Hacibe Hallal' ${ }^{3}$, German Soriano ${ }^{4}$, \\ Eva Roman 4,5, Francisco Ruiz-Cabello3, Manuel Romero-Gomez ${ }^{6}$, M. Isabel Lucena ${ }^{1 *}$ \\ and Raúl J. Andrade ${ }^{1}$
}

\footnotetext{
' Unidad de Gestión Clínica de Aparato Digestivo, Servicio de Farmacología Clínica, Instituto de Investigación Biomédica de Málaga, Hospital Universitario Virgen de la Victoria, Universidad de Málaga, CIBERehd, Málaga, Spain, ${ }^{2}$ Unidad de Gestión Clínica de Laboratorio, Departamento de Bioquímica y Biología Molecular III/Inmunología, Instituto de Investigación Biosanitario de Granada, Complejo Hospitalario de Granada, Universidad de Granada, Granada, Spain, ${ }^{3}$ Servicio de Aparato Digestivo, Hospital Morales Meseguer, Murcia, Spain, ${ }^{4}$ Servicio de Gastroenterología, Hospital de la Santa Creu i Sant Pau, Universitat Autònoma de Barcelona, CIBERehd, Barcelona, Spain, ${ }^{5}$ Escola Universitària d'Infermeria-Sant Pau, Universitat Autònoma de Barcelona, Barcelona, Spain, ${ }^{6}$ Unidad de Gestión Clínica de Aparato Digestivo Intercentros, Hospitales Universitarios Virgen Macarena-Virgen del Rocio, CIBERehd, Seville, Spain
}

Natural killer cells are an integral part of the immune system and represent a large proportion of the lymphocyte population in the liver. The activity of these cells is regulated by various cell surface receptors, such as killer lg-like receptors (KIR) that bind to human leukocyte antigen (HLA) class I ligands on the target cell. The composition of $\mathrm{KIR}$ receptors has been suggested to influence the development of specific diseases, in particularly autoimmune diseases, cancer and reproductive diseases. The role played in idiosyncratic drug-induced liver injury (DILI) is currently unknown. In this study, we examined KIR gene profiles and HLA class I polymorphisms in amoxicillin-clavulanate (AC) DILI patients in search for potential risk associations. One hundred and two AC DILI patients and 226 controls were genotyped for the presence or absence of $16 \mathrm{KIR}$ loci, including the two pseudogenes 2DP1 and 3DP1. No significant differences were found in the distribution of individual KIRs between patients and controls, which were comparable to previously reported KIR data from ethnically similar cohorts. The 21.6 and $21.2 \%$ of the patients and controls, respectively, were homozygous haplotype $A$ carriers, while 78.4 and $78.8 \%$, respectively, contained at least one B haplotype (Bx). The genotypes translated into 27 (AC DILI) and 46 (controls) different gene profiles, with 19 being present in both groups. The most frequent $\mathrm{Bx}$ gene profile containing KIRs 2DS2, 2DL2, 2DL3, 2DP1, 2DL1, 3DL1, 2DS4, 3DL2, 3DL3, 2DL4, and 3PD1 was present in $16 \%$ of the DILI patients and $14 \%$ of the controls. The distribution of HLA class I epitopes did not differ significantly between AC DILI patients and controls. The most frequent receptor-ligand combinations in the DILI patients were 2DL3 + epitope C1 (67\%) and 3DL1 + Bw4 motif (67\%), while 2DL1 + epitope C2 (69\%) 
and 3DL1 + Bw4 motif (69\%) predominated in the controls. This is to our knowledge the first analysis of KIR receptor-HLA ligand associations in DILI, although our findings do not support evidence of these genetic variations playing a major role in AC DILI development.

Keywords: hepatotoxicity, drug-induced liver injury, pharmacogenetics, immune response, HLA, receptor/ligand

\section{INTRODUCTION}

Idiosyncratic drug-induced liver injury (DILI) is a condition that symptomatically can mimic most kinds of acute and chronic liver disorders. Unlike intrinsic DILI, it cannot be predicted based on drug pharmacological action or dose. It is believed that idiosyncratic DILI occurs in one of every 10000 to 100 000 patients taking a specific medication, with some drugs being more prone to cause DILI than others. The majority of idiosyncratic DILI cases fully recuperates after drug cessation, while $4-10 \%$ go on to develop acute liver failure (Björnsson and Olsson, 2005; Robles-Diaz et al., 2014). Furthermore, DILI is a major factor in drug development discontinuations and postmarketing drug withdrawals. Despite increased interest, the cellular mechanism behind DILI is not fully understood. Previous studies have, however, demonstrated an apparent role for the immune system in various forms of idiosyncratic DILI, including higher representation of specific HLA class I and II alleles in amoxicillin-clavulanate (AC) DILI patients (Lucena et al., 2011; Stephens et al., 2013; Grove and Aithal, 2015).

Natural killer (NK) cells are an integral part of the innate immune system, in particularly in the liver, with cytolytic capacity to kill cancer and infected cells. Furthermore, NK cells can contribute to inflammation and immunoregulation through their release of cytokines and subsequently also play a modifying role in adaptive immunity. The activity of NK cells is regulated by various types of cell surface receptors, such as killer Iglike receptors (KIR). The KIR family contains both inhibitory (iKIR: 2DL1, 2DL2, 2DL3, 2DL4, 2DL5, 3DL1, 3DL2, and 3DL3) and activating (aKIR: 2DS1, 2DS2, 2DS3, 2DS4, 2DS5, and 3DS1) receptors. Although initially believed to be an inhibitory receptor, KIR2DL4 is now considered to have both inhibitory and activating signaling domains (Moradi et al., 2015). The KIR genes are highly variable, with polymorphisms on gene as well as allele levels. Both aKIRs and iKIRs are expressed on the same NK cell and the exact KIR composition varies from individual to individual. The KIR genes are generally inherited as haplotype A or B. Group A comprises six iKIRs (2DL1, 2DL3, 2DL4, 3DL1, 3DL2, and 3DL3), one aKir (2DS4) and two pseudogenes (2DP1 and 3DP1). Any other KIR gene combination with at least one of the KIR2DL2, 2DL5, 3DS1, 2DS1, 2DS2, $2 D S 3$, or $2 D S 5$ genes present, is referred to as group $B$ haplotype (Uhrberg et al., 1997). The balance of activating and inhibitory KIRs may subsequently contribute to enhanced risk of distinct diseases.

Inhibitory NK cell activity signals are mainly induced through the recognition of HLA class I ligands by iKIR receptors. KIR2DL1 binds preferentially to HLA-C allotypes characterized by a lysine in amino acid position 80 (C2 epitope), while
KIR2DL3 and particularly 2DL2 have higher recognition affinity for the $\mathrm{C} 1$ epitope, containing an asparagine in the same position (Winter and Long, 1997; Moesta et al., 2008). The HLA-B alleles can be divided into allotypes containing the serological Bw4 or Bw6 public epitope, with the exception of a few B alleles that do not contain either of these epitopes (Gumperz et al., 1997). KIR3DL1 binds preferentially to HLA-B allotypes characterized by the Bw4 epitope, which is defined by the amino acid motifs at positions 77-83. The HLA-A alleles $A^{*} 23: 01, A^{*} 24: 02$, and $A^{*} 32: 01$ have also been found to contain the Bw4 epitope and subsequently can act as ligands for KIR3DL1. The KIR3DL2 receptor has been shown to bind to HLA-A3 and A11 allotypes, although the interaction appears to be highly dependent on the peptide bound to the HLA complex (Hansasuta et al., 2004). In addition, a recent study has demonstrated that HLA-B27 can also interact with KIR3DL2, although B27 is less frequent than the HLA-A allotypes in most populations (Shaw et al., 2014). The aKIR receptor ligands are less well established. It is believed that many aKIR receptors share HLA ligands with their corresponding inhibitory counterparts, but bind with lower affinity (Stewart et al., 2005).

The existing variability in KIR and HLA profiles between individuals could potential result in specific profiles being more prone to certain medical conditions, as suggested for Italian KIR2DS1 and HLA-C2 ligand carriers being more susceptible to autoimmune hepatitis (Littera et al., 2016). We have previously demonstrated that HLA alleles have an effect on AC DILI susceptibility and phenotype. As HLA class I alleles are involved in the regulation of NK cell activity through KIR receptors, and considering the increased abundance of NK cells in the liver, we aimed to evaluate the contribution of HLA class I profiles in conjunction with KIR gene repertoires to determine potential genetic predispositions for AC DILI in Spanish subjects.

\section{MATERIALS AND METHODS}

\section{Subjects and Study Protocol}

A total of 102 AC DILI cases were selected from those submitted to the Spanish DILI Registry, a collaborative network established in 1994 to prospectively identify cases of DILI in a standardized manner. The criteria for DILI at the time of subject inclusions in the study were: an increase in alanine transaminase (ALT) $\geq 3$ times the upper limit of normal (xULN) or $\geq 2 x U L N$ of alkaline phosphatase (ALP) or total bilirubin (TBL) $\geq 2 \times U L N$ if associated with elevations of ALT or ALP. However, 91\% of the cases also fulfilled the more recent DILI criteria established by Aithal et al. (2011): ALT $\geq 5 \mathrm{xULN}$ or ALT $\geq 3 \mathrm{xULN}+$ $\mathrm{TBL} \geq 2 \mathrm{xULN}$ or ALP $\geq 2 \mathrm{xULN}$. The pattern of liver injury was 
classified based on $R$-value calculations as previously described (Aithal et al., 2011). A detailed description of the operational structure of the registry, data recording and case ascertainment has been reported elsewhere (Andrade et al., 2005). As a control group, we selected 226 unrelated Spanish subjects of Caucasian descent, without previous DILI history. These controls were recruited from the Spanish Bone Marrow Donor Registry as well as hospital volunteers from the same geographic region and of similar age and gender distribution. Due to the frequent use of $\mathrm{AC}$ in the general population in Spain it was assumed that a large proportion of the controls would have taken AC during some stage of their lives without developing DILI.

\section{Ethics Statement}

The study protocol was approved by the local ethics committee of the coordinating center at the Hospital Universitario Virgen de la Victoria (Comité Ético de Investigación) in Málaga, Spain. All subjects who took part in the study were 18 years of age or older. All subjects gave written informed consent, which conformed with the current Helsinki Declaration, prior to study enrolment.

\section{DNA Extraction and Genotyping}

Venous blood was obtained from each subject and DNA was extracted using QIAamp DNA Blood Mini Kit (QIAGEN, Hilden, Germany) according to the manufacturer's instructions. Genotyping of KIRs 2DL1, 2DL2, 2DL3, 2DL4, 2DL5, 3DLI, 3DL2, 3DL3, 2DS1, 2DS2, 2DS3, 2DS4, 2DS5, 3DS1, 2DP1, and 3DP1 was performed using the Lifecodes KIR-SSO typing Kit 545110R (Immucor Inc, Norcross, GA, USA) and analyzed with a BioPlex 200 system (Luminex xMAP, Austin, TX, USA) according to the manufacturer's instructions. Haplotype designation was determined as previously described (Uhrberg, 2005). Individuals carrying only haplotype A genes (KIR2DL1, 2DL3, 2DL4, 3DL1, $3 D L 2,3 D L 3,2 D S 4,2 D P 1$, and $3 D P 1)$ were considered as AA genotype carriers. All other KIR gene combinations were referred to as Bx genotypes, where $\mathrm{x}$ indicates an $\mathrm{A}$ or $\mathrm{B}$ haplotype.

Genotyping of the HLA class I (A, B, and C) loci was performed using the LABType ${ }^{\circledR}$ SSO typing test (One Lambda, Canoga Park, CA, USA). Target DNA was amplified by PCR using sequence-specific primers, followed by hybridization with allele-specific oligodeoxynucleotides coupled with fluorescent phycoerythrin-labeled microspheres. The fluorescence intensity was determined with a Bio-Plex 200 system (Luminex xMAP, Austin, TX, USA). HLA alleles were assigned using the HLATools software (One Lambda, Canoga Park, CA, USA). The HLA ligand groups were assigned based on the amino acid residues of the corresponding alleles. The HLA-C alleles containing an asparagine at position 80 were considered as being $\mathrm{C} 1$ and those with a lysine in the same position as C2. Similarly, the HLA$\mathrm{B}$ alleles were classified as HLA-Bw4 and Bw6 depending on the amino acids at positions $77-83^{1}$. In addition, HLA-A*23:01, $A^{*} 24: 02$, and $A^{*} 32: 01$ were also considered as Bw4 containing alleles based on previously demonstrated results (Stern et al., 2008).

\footnotetext{
${ }^{1}$ http://www.ufrgs.br/imunovet/molecular_immunology/hla.html
}

\section{Statistical Analysis}

Human leukocyte antigen allele distributions were compared between DILI patients and controls using a comparison of proportions test, a derivative of Fisher's exact test, and $p<0.05$ was considered to be statistically significant.

\section{RESULTS}

\section{DILI Patient Characteristics}

A total of 102 AC DILI patients were included in the study of which 60 were males and 42 females. The mean age at DILI onset was 59 years, ranging from 18 to 88 years. The predominant pattern of injury, based on the $R$-value calculated from the first blood sample analysis after DILI recognition, was hepatocellular $(n=39)$ followed by mixed $(n=34)$ and cholestatic $(n=29)$. Of the 102 patients two developed liver failure resulting in death or liver transplantation. Demographics, clinical, and laboratory parameters of the 102 DILI cases included in the study are outlined in Table 1.

\section{KIR Gene Distribution}

All the 102 AC DILI patients and 226 controls were genotyped for the presence or absence of $16 \mathrm{KIRs}$, including the two pseudogenes 2DP1 and 3DP1. No significant differences were found in the distribution of individual KIR genes between patients and controls. The four framework genes KIR3DL2, 3DL3, $3 D P 1$, and $2 D L 4$ were present in all tested subjects. KIR2DL1, $3 D L 1,2 D S 4$, and 2DP1 were found in more than $90 \%$ of both the patient and control cohorts, while 2DS1, 2DS3, 2DS5, and 3DS1 where the least present genes, only seen in less than $45 \%$ of the

TABLE 1 | Demographics, clinical, and laboratory parameters of the 102 DILI cases included in the study.

\begin{tabular}{lc} 
Demographics & \\
Mean age, years (range) & $59(18-88)$ \\
Male/female, $n$ & $60 / 42$ \\
Body mass index, mean $\pm \mathrm{SD}$ & $26.1 \pm 3.2$ \\
Time to DILI onset, $\boldsymbol{n}$ & 62 \\
$<15$ days & 26 \\
$15-30$ days & 14 \\
$>30$ days & \\
Clinical presentation, $\boldsymbol{n}$ & 85 \\
Jaundice & 59 \\
Hospital admission & \\
Type of liver injury, $\boldsymbol{n}$ & 39 \\
Hepatocellular & 29 \\
Cholestatic & 34 \\
Mixed & \\
Biochemical parameters*, mean \pm SD (median) & $7.6 \pm 5.5(6.7)$ \\
Total bilirubin (mg/dL) & $18.0 \pm 19.4(9.8)$ \\
ALT xULN, hepatocellular and mixed cases & $2.7 \pm 1.6(2.2)$ \\
ALP xULN, cholestatic and mixed cases & \\
\hline *First available serum analysis after DILI onset. ALT, alanine transaminase; ALP, \\
alkaline phosphatase; ULN, upper limit of normal.
\end{tabular}

\section{Demographics}

Mean age, years (range)

Body mass index, mean \pm SD

Time to DILI onset,

$<15$ days

62

26

Type of liver injury, $\boldsymbol{n}$

Hepatocellular

39

29

Mixed

$7.6 \pm 5.5(6.7)$

$18.0 \pm 19.4(9.8)$ alkaline phosphatase; ULN, upper limit of normal. 
subjects (Table 2). While the KIR2DS4 gene was among the more frequently detected genes, 46 and $51 \%$ of the KIR2DS4 carrying DILI patients and controls, respectively, were homozygous for a 22 base pair deletion, which produces a non-functional receptor, and $37 \%$ in both subgroups were found to be heterozygous for the same mutation.

Among the DILI cases $21.6 \%$ were homozygous for the A haplotype (AA genotype) while $78.4 \%$ contained a B haplotype (Bx: $\mathrm{AB}$ or $\mathrm{BB}$ genotypes). The corresponding genotype distribution in the control subjects were 21.2 and $78.8 \%$, respectively. Hence, no significant difference in genotype distribution was found between the two groups. The determined genotypes translated into 27 different gene profiles in the DILI patients and 46 in the controls. Nineteen of the gene profiles were present in both groups, while 8 were specific to the DILI patients and 27 to the controls (Figure 1). The individual DILI-specific genotypes demonstrated frequencies of $0.1-2.0 \%$, while the individual control-specific genotypes had frequencies of $0.4-2.2 \%$. Despite the relatively low number of 'group-specific' genotypes, the DILI group presented a significantly smaller proportion of individuals with DILI-specific genotypes compared to that of controls with control-specific genotypes ( $8.8 \%$ vs. $19 \%$, $p=0.024)$.

None of the individual Bx genotypes present in both groups were found to be significantly different with respect to frequency in the DILI group compared to the controls. The most frequent gene profile among the $\mathrm{Bx}$ haplotype carriers contained $3 D L 1$, $2 D L 1,2 D L 3,2 D S 4,2 D L 2,2 D S 2$, and $2 D P 1$ in addition to the four framework loci, and was present in $15.7 \%$ of the DILI patients and $14.2 \%$ of the controls (Figure 1). Of the AA haplotype carriers with $2 S D 4$ as the only activating receptor, $54.5 \%$ of the DILI patients and $60.4 \%$ of the controls where homozygous for the 22 base pair deletion in the $2 S D 4$ gene, which subsequently lead to a haplotype without any aKIR receptor. In addition, 45.5 and 35.4\% of the DILI and control AA haplotype carriers, respectively, were found to be heterozygous for same deletion.

Activating KIR genes have been associated with increased susceptibility to certain diseases, in particular autoimmune related disorders. Hence, we compared DILI patients and controls with respect to the total number of aKIR genes in each subject. KIR2DL4 was not included in this analysis, as it was present in all study subjects and is believed to have both inhibitory and activating functions. Furthermore, presence of the 22 bp deletion in exon 5 causing a truncated KIR2DS4 protein was taken into consideration. Hence, subjects homozygous for the deletion allele and with no other aKIR genes were considered as having no aKIRs. One single aKIR gene was most frequently seen among both DILI patients (21.6\%) and controls (20.8\%), followed by four aKIR genes in the DILI (16.7\%) and five aKIR genes in the control group (16.4\%). The presence of six aKIR genes occurred most infrequently in both the groups, although the proportion was slightly higher among the DILI patients $(4.9 \%$ vs. $2.6 \%)$. No statistically significant differences were found comparing DILI patients and controls classified by total number of aKIR genes per subject (Figure 2).

To determine any potential effects of KIR genes on disease phenotype, the presence of KIR genes was also analyzed in the 


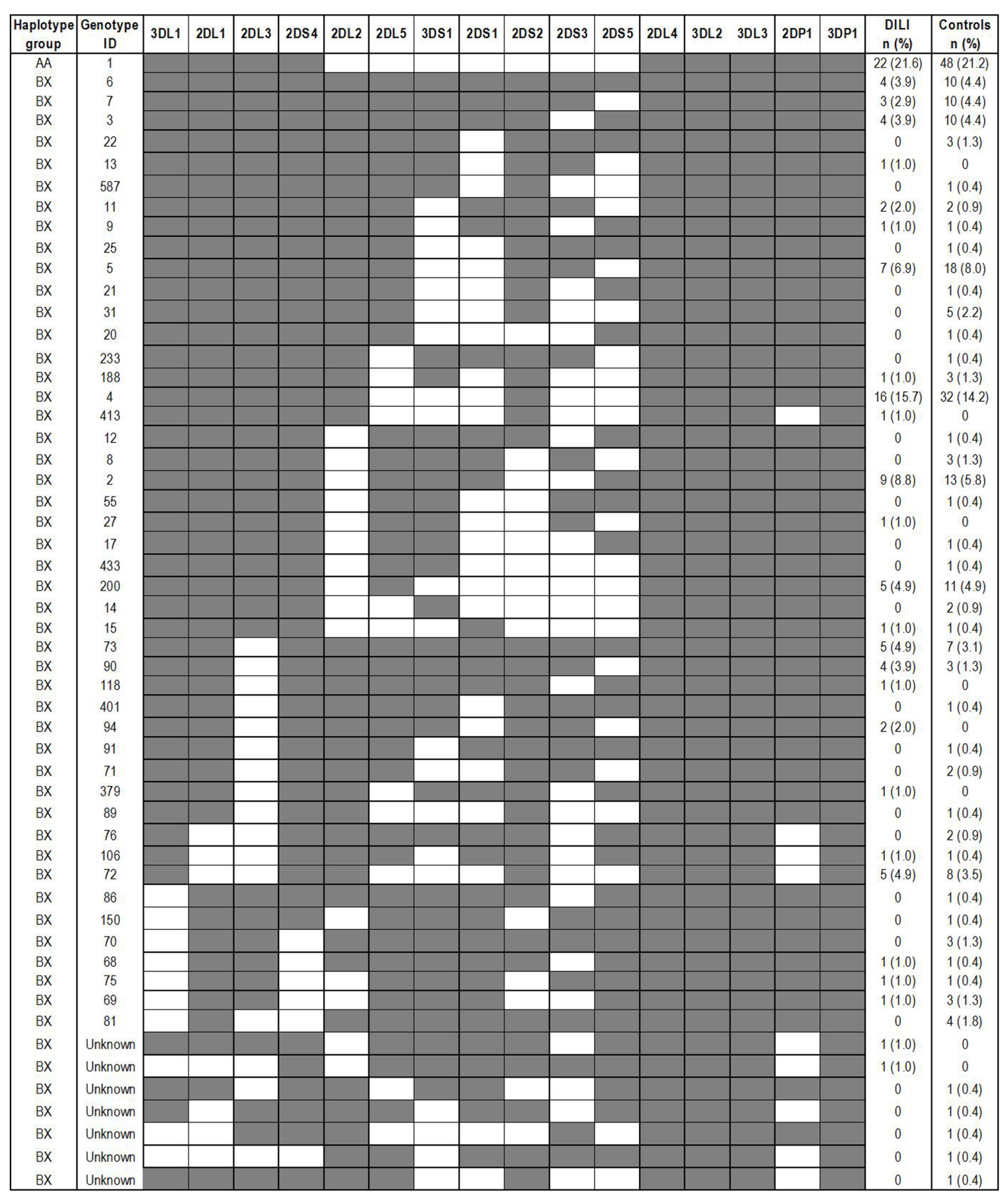

FIGURE 1 | KIR genotype distribution in 102 Spanish amoxicillin-clavulanate-induced liver injury patients and 226 controls. Genotype IDs refer to ID numbers assigned by the Allele Frequency Net Database (www.allelefrequencies.net). The presence and absence of a KIR gene are indicated by a shaded and white box, respectively.

AC DILI cohort stratified by pattern of liver injury. No significant differences were found in the distribution of individual KIR genes between patients presenting with hepatocellular, cholestatic and mixed type of liver injury compared with the controls (data not shown).

\section{HLA Ligand Distribution}

All patients and controls were genotyped for the HLA class I loci $(\mathrm{A}, \mathrm{B}$, and $\mathrm{C})$ in order to determine the distribution of epitopes $\mathrm{C} 1, \mathrm{C} 2$, and Bw4 as well as the allele groups A3/A11 and B27, which act as ligands for different KIR receptors. Phenotype 


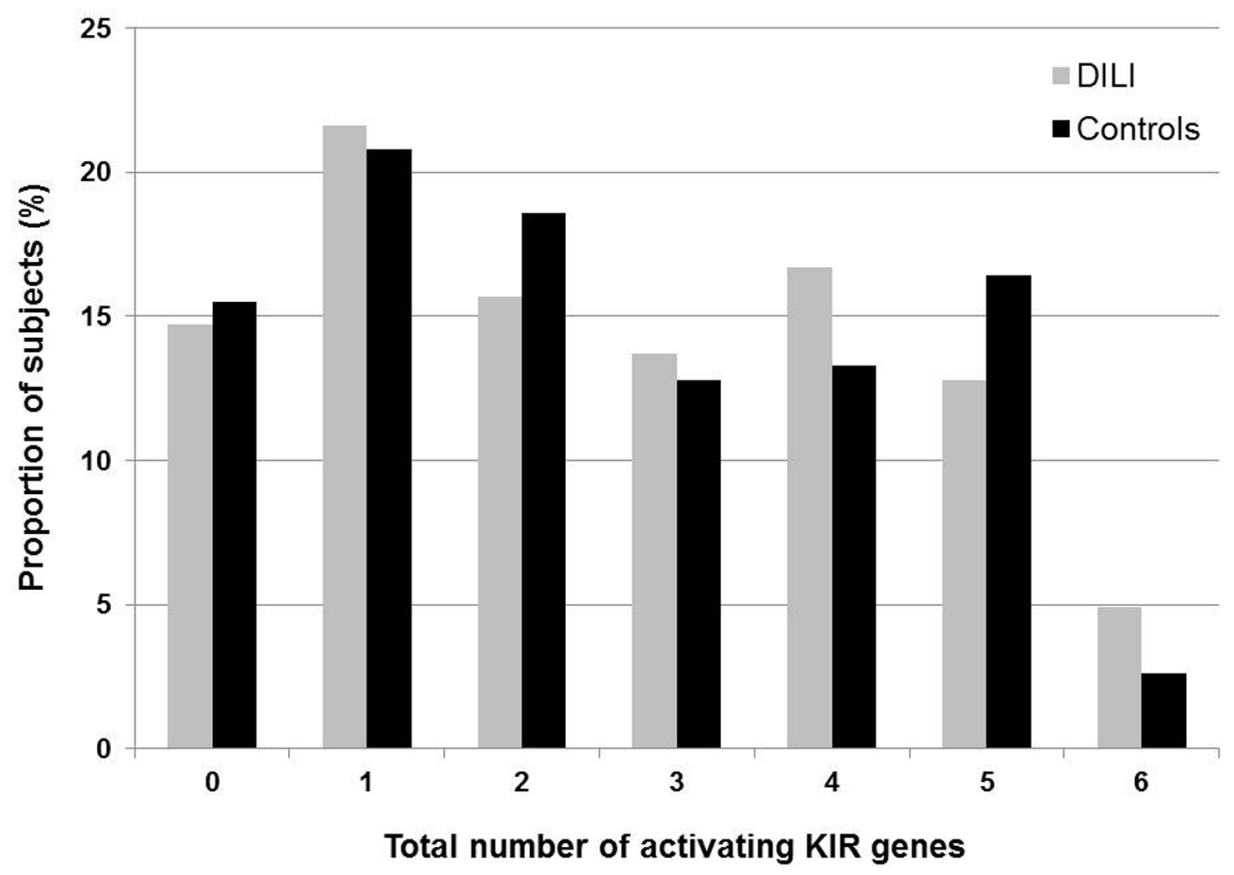

FIGURE 2 | Total number of activating KIR genes (2DS1, 2DS2, 2DS3, 2DS4, 2DS5, and 3DS1) present in 102 Spanish amoxicillin-clavulanate-induced liver injury patients and $\mathbf{2 2 6}$ controls. Only full-length 2DS4 is considered as an activating KIR, while the presence of 2DS4 with a 22 base pair deletion in exon 5 causing a truncated KIR2DS4 is not.

distributions of the HLA ligands are presented in Table 3. HLA $\mathrm{C} 1$ was more frequent than $\mathrm{C} 2$ and in both DILI patients $(83 \%$ vs. $66 \%$ ) and controls ( $78 \%$ vs. $73 \%$ ), though the difference was more pronounced among the DILI patients. Sixty percent of the DILI patients contained at least one copy of the HLA-B Bw4 motif compared to $65 \%$ in the controls. The distribution of Bw4Thr80 and Bw4-Ile80 motifs was relatively equal with $36 \%$ vs. $33 \%$ in the DILI patients and $37 \%$ vs. $38 \%$ in the control subjects, respectively. The proportion of Bw4 containing HLA-A allele carriers was slightly higher in the control subject compared to

TABLE 3 | Phenotype distribution of HLA class I KIR ligands in 102 Spanish amoxicillin-clavulanate-induced liver injury patients and 226 controls.

\begin{tabular}{lcc}
\hline HLA class I & DILI, $\boldsymbol{n ( \% )}$ & Controls, $\boldsymbol{n}(\%)$ \\
\hline HLA-C1 & $85(83)$ & $176(78)$ \\
HLA-C2 & $67(66)$ & $164(73)$ \\
C1/C1 & $35(34)$ & $62(27)$ \\
C2/C2 & $17(17)$ & $50(22)$ \\
C1/C2 & $50(49)$ & $114(50)$ \\
HLA-B Bw4 & $61(60)$ & $147(65)$ \\
HLA-B Bw4/Bw4 & $18(18)$ & $44(19)$ \\
HLA-B Bw4-Thr80 & $36(35)$ & $83(37)$ \\
HLA-B Bw4-Ile80 & $34(33)$ & $85(38)$ \\
HLA-A Bw4* & $18(18)$ & $60(27)$ \\
HLA-A3 or A11 & $32(31)$ & $59(26)$ \\
HLA-B27 & $4(3.9)$ & $13(5.8)$ \\
\hline
\end{tabular}

*HLA-A*23:01, 24:02, and 32:01.
DILI patients (27\% vs. $18 \%$ ), while $31 \%$ of the DILI patients contained HLA-A3 or A11 alleles compared to $26 \%$ of the controls. In addition to A3 and A11, B27 has also been reported to be a potential KIR3DL2 ligand. The distribution of B27 carriers was comparable between the two study populations $(3.9 \%$ vs. $5.8 \%)$. None of the analyzed ligands differed significantly with respect to carrier distributions between DILI cases and controls.

\section{KIR Receptor-HLA Ligand Distributions}

The KIR receptors bind to specific HLA ligands. Hence the combination of KIR genes and corresponding HLA ligands present in the subjects was also analyzed. The most frequently found receptor-ligand combinations in the DILI patients were $2 \mathrm{DL} 3+\mathrm{C} 1(67 \%)$ and 3DL1 + Bw4 motif (67\%), while 2DL1 + C2 (69\%) and 3DL1 + Bw4 motif (69\%) predominated in the controls. In contrast, 2DS4 with at least one copy of the full length gene combined with HLA A11 alleles was the least frequently found receptor-ligand combination in both the DILI (3.9\%) and the control (4.9\%) group. No significant differences in phenotype distribution of the analyzed KIR gene and HLA ligand combinations were found between DILI patients and controls (Table 4).

\section{DISCUSSION}

Natural killer cells are enriched in the liver, comprising 25-40\% of intrahepatic lymphocytes in normal adult livers (Tian et al., 2013). While these cells play an important role in liver diseases 
TABLE 4 | Phenotype distribution of KIR genes and HLA ligands in 102 Spanish DILI patients and 226 controls.

\begin{tabular}{lcc}
\hline KIR + HLA ligands, $\boldsymbol{n}(\%)$ & DILI, $\boldsymbol{n}(\%)$ & Controls, $\boldsymbol{n}(\%)$ \\
\hline Inhibitory & & \\
2DL1 + C2 & $63(62)$ & $156(69)$ \\
2DL2 + C1 & $51(50)$ & $108(48)$ \\
2DL3 + C1 & $68(67)$ & $150(66)$ \\
3DL1 + Bw4* & $68(67)$ & $157(69)$ \\
3DL1 + Bw4 (A) & $57(25)$ \\
3DL1 + Bw4 (B) & $18(18)$ & $135(60)$ \\
3DL1 + Bw4 Thr80 & $58(57)$ & $77(34)$ \\
3DL1 + Bw4 lle80 & $34(33)$ & $77(34)$ \\
3DL2 + A3/A11/B27 & $32(31)$ & $66(29)$ \\
Activating & $36(35)$ & \\
2DS1 + C2 & & $61(27)$ \\
2DS2 + C1 & $27(26)$ & $106(47)$ \\
2DS2 + A11 & $53(52)$ & $23(10.2)$ \\
2DS4 + A11 & $6(5.9)$ & $11(4.9)$ \\
3DS1 + Bw4 motif* & $4(3.9)$ & $62(27)$ \\
3DS1 + Bw4 (A) & $27(26)$ & $25(11)$ \\
3DS1 + Bw4 (B) & $9(9)$ & $52(23)$ \\
3DS1 + Bw4 Thr80 & $22(22)$ & $30(13)$ \\
3DS1 + Bw4 lle80 & $12(12)$ & $30(13)$ \\
\hline
\end{tabular}

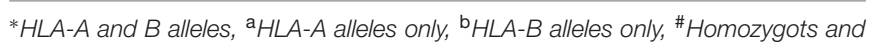
hetrozygots for the full length allele only.

by inhibiting viral infection, tumor cell growth and liver fibrosis, they can also be harmful causing hepatocellular damage and inhibiting liver regeneration if not controlled properly. Impaired regulation of NK cell activity is thought to contribute to hepatic diseases such as autoimmune hepatitis, primary biliary cirrhosis and sclerosing cholangitis (Tian et al., 2013). The fact that intrahepatic NK cells exhibit higher level of cytotoxicity and increased expression of cytotoxic mediators compared to peripheral NK cells highlights the importance of adequate regulation of NK cell activity (Ishiyama et al., 2006). Due to the crucial role of KIR receptors in NK cell activity regulation, KIR genotype analyses have been performed across various conditions, including hepatic diseases, to elucidate the potential contribution of KIR variability in disease susceptibility. In this study, we have analyzed KIR and HLA polymorphisms with regards to AC DILI susceptibility. This is to our knowledge the first KIR analysis focusing on DILI. We did not find any significant differences in the frequency of individual KIR genes between DILI cases and controls. Furthermore, carrier frequencies in the current control group were similar to those of independent Spanish subjects previously published (Hollenbach et al., 2010). One must also keep in mind that not only the KIR gene repertoire varies between individuals but that each KIR gene is also highly polymorphic. There are currently 753 different KIR alleles in total present in the IPD-KIR database (Robinson et al., 2005). Hence, analyzing only presence or absence of KIR genes could obscure existing allele associations.

The presence of aKIRs is generally more variable than that of iKIRs. It has been suggested that the total number of aKIRs could affect disease susceptibility as augmented signals from multiple
aKIR receptors might exacerbate the activation of NK cells and $\mathrm{T}$ cell subsets against self-antigens leading to disease conditions (Ashouri et al., 2014). Hence, we compared the total number of aKIRs in individual DILI patients and controls, but did not find any significant differences in the proportion of subjects classified by aKIRs, ranging from 0 to 6 different types of activating receptors. As associations between dominant aKIR repertoires and disease susceptibility detected to date are mainly limited to autoimmune conditions, such as lupus erythematosus, VogtKoyanagi-Harada syndrome, multiple sclerosis, Hashimoto's thyroiditis and autoimmune hepatitis (Hou et al., 2010; Levinson et al., 2010; García-León et al., 2011; Ashouri et al., 2014; Littera et al., 2016) a dominance of aKIR receptors may be less important in AC DILI. Nevertheless, autoimmune conditions can also be negatively associated with aKIRs as demonstrated for pemphigus foliaceus. Here the presence of more than three aKIR genes appears to play a protective role, which highlights the fact that the role of aKIR dominance is not general but dependent on the underlying mechanism of the condition in question (Augusto et al., 2012).

Similar to the lack of association between aKIRs and DILI susceptibility, the proportions of $\mathrm{AA}$ and $\mathrm{Bx}$ genotype carriers were comparable between patients and controls. AA1 was the most frequent genotype in both groups. The $\mathrm{Bx}$ genotypes translated to 26 and 45 different genotypes in the DILI patients and controls, respectively, with $18 \mathrm{Bx}$ genotypes being present in both groups with similar frequencies. The three most frequent $\mathrm{Bx}$ genotypes in both the DILI and control group were $\mathrm{Bx} 4$, $\mathrm{Bx} 5$, and $\mathrm{Bx} 2$, which together made up $40 \%$ and $35 \%$ of the $\mathrm{Bx}$ genotype carriers in the two populations, respectively. The individual genotype distributions of $\mathrm{Bx} 4, \mathrm{Bx} 5$, and $\mathrm{Bx} 2$ were similar to those previously observed in different Spanish and other European populations ${ }^{2}$. These three genotypes are also among the most common KIR genotypes worldwide (Hollenbach et al., 2012). In the current study, we found nine Bx genotypes that were specific to the DILI patients and 27 that were only found in the control groups. However, it is unlikely that the DILIspecific genotypes would have any bigger impact on AC DILI susceptibility due to the small number of patients that were found to be carriers of these KIR genotypes. Similarly, the low frequency of the individual control-specific genotypes does not speak in favor of these genotypes having any protective effect against AC DILI.

The function of KIR receptors depends on the presence of appropriate HLA ligands on the target cell in order for correct signaling and NK cell activity to occur. Hence, we analyzed the presence of currently identified HLA class I ligands in our patient and control cohorts but did not find any significant differences between the two groups. HLA ligands have, however, been associated with liver disease progression. For example, HLA-C1 homozygosity and presence of the HLA-Bw4 I80 variant have been associated with better overall survival of hepatitis $\mathrm{C}$ virus-related hepatocellular carcinoma after curative treatment (Cariani et al., 2013), while HLA-C2 homozygosity has been associated with increased risk of hepatitis $\mathrm{C}$ virus infection

\footnotetext{
${ }^{2}$ www.allelefrequencies.net
} 
treatment failure (Suppiah et al., 2011). Evidence of enhanced risk of developing primary sclerosing cholangitis in HLA-Bw6 and HLA-C1 homozygotes has also been presented (Karlsen et al., 2007).

Considering KIR and HLA repertoires simultaneously has likewise been explored in search for the role of NK cells in disease conditions. Our analysis did not reveal any significant difference in concurrent KIR receptor and HLA ligand carriage between DILI patients and control subjects. The KIR-HLA combinations considered in this study were based on identified ligands to date. However, the current understanding of KIR ligands is not complete and presently unknown KIR receptor-HLA ligand interactions could possibly influence DILI susceptibility and should therefore not be overlooked. Furthermore, it has been suggested that different KIR allele products can have different affinity for the same HLA ligand (Frazier et al., 2013), which could lead to differences in NK cell activity regulation. As our KIR analysis was limited to presence or absence of KIR receptors, this aspect was consequently not evaluated.

In summary, in the first exploratory study of 102 Spanish AC DILI cases and 226 controls no significant differences in KIR repertoires and corresponding HLA class I ligand profiles were detected. These results are suggestive of KIRs not being major risk factors for AC DILI susceptibility. However, as the mechanism of KIR receptors is not completely elucidated to date and NK cell activity regulation systems additional to KIR are

\section{REFERENCES}

Aithal, G. P., Walkins, P. B., Andrade, R. J., Larrey, D., Mookhia, M., Takikawa, H., et al. (2011). Case definition and phenotype standardization in drug-induced liver injury. Clin. Pharmacol. Ther. 89, 806-815. doi: 10.1038/clpt.2011.58

Andrade, R. J., Lucena, M. I., Fernández, M. C., Pelaez, G., Pachkoria, K., GarcíaRuiz, E., et al. (2005). Drug-induced liver injury: an analysis of 461 incidences submitted to the Spanish registry over a 10-year period. Gastroenterology 129, 512-521. doi: 10.1053/j.gastro.2005.05.006

Ashouri, E., Dabbaghmanesh, M. H., and Ranjbar Omrani, G. (2014). Presence of more activating KIR genes is associated with Hashimoto's thyroiditis. Endocrine 46, 519-525. doi: 10.1007/s12020-013-0080-2

Augusto, D. G., Lobo-Alves, S. C., Melo, M. F., Pereira, N. F., and Petzl-Erler, M. L. (2012). Activating KIR and HLA Bw4 ligands are associated to decreased susceptibility to pemphigus foliaceus, an autoimmune blistering skin disease. PLoS ONE 7:e39991. doi: 10.1371/journal.pone.0039991

Björnsson, E., and Olsson, R. (2005). Outcome and prognostic markers in severe drug-induced liver disease. Hepatology 42, 481-489. doi: 10.1002/hep.20800

Cariani, E., Pilli, M., Zerbini, A., Rota, C., Olivani, A., Zanelli, P., et al. (2013). HLA and killer immunoglobulin-like receptor genes as outcome predictors of hepatitis C virus-related hepatocellular carcinoma. Clin. Cancer Res. 19, 5465-5473. doi: 10.1158/1078-0432.CCR-13-0986

Frazier, W. R., Steiner, N., Hou, L., Dakshanamurthy, S., and Hurley, C. K. (2013). Allelic variation in KIR2DL3 generates a KIR2DL2-like receptor with increased binding to its HLA-C ligand. J. Immunol. 190, 6198-6208. doi: 10.4049/jimmunol.1300464

García-León, J. A., Pinto-Medel, M. J., García-Trujillo, L., López-Gómez, C., Oliver-Martos, B., Prat-Arrojo, I., et al. (2011). Killer cell immunoglobulinlike receptor genes in Spanish multiple sclerosis patients. Mol. Immunol. 48, 1896-1902. doi: 10.1016/j.molimm.2011.05.018

Grove, J. I., and Aithal, G. P. (2015). Human leukocyte antigen genetic risk factors of drug-induced liver toxicology. Expert. Opin. Drug Metab. Toxicol. 11, 395-409. doi: 10.1517/17425255.2015.992414

Gumperz, J. E., Barber, L. D., Valiante, N. M., Percival, L., Phillips, J. H., Lanier, L. L., et al. (1997). Conserved and variable residues within the Bw4 motif present, a potential role for NK cells activity in DILI should not be completely dismissed. Further studies on the level of KIR receptor expression and cytokine production are warranted in this area.

\section{AUTHOR CONTRIBUTIONS}

Study concept and design (CS, RA, and ML); patient recruitment and data acquisition (MG-C, IM-C, HH, GS, ER, and MR-G); performed experiments (AM-C and M-AL-N) analysis and interpretation of data (CS, M-AL-N, FR-C, RA, and ML); drafting of the manuscript (CS, RA, and ML); critical revision of the manuscript (MG-C, M-AL-N, and FR-C).

\section{FUNDING}

The present study has been supported by grants of the Instituto de Salud Carlos III co-founded by Fondo Europeo de Desarrollo Regional - FEDER (contract numbers: PI12/00378, SAS-PI0239/2012, AC-0073-2013) and by the Agencia Española del Medicamento. CIBERehd is funded by Instituto de Salud Carlos III. The funding sources had no involvement in the study design; in the collection, analysis, and interpretation of data; in the writing of the report or in the decision to submit the manuscript for publication.

of HLA-B make separable contributions to recognition by the NKB1 killer cell-inhibitory receptor. J. Immunol. 158, 5237-5241.

Hansasuta, P., Dong, T., Thananchai, H., Weekes, M., Willberg, C., Aldemir, H., et al. (2004). Recognition of HLA-A3 and HLA-A11 by KIR3DL2 is peptidespecific. Eur. J. Immunol. 34, 1673-1679. doi: 10.1002/eji.200425089

Hollenbach, J. A., Meenagh, A., Sleator, C., Alaez, C., Bengoche, M., Canossi, A., et al. (2010). Report from the killer immunoglobulin-like receptor (KIR) anthropology component of the 15th international histocompatibility workshop: worldwide variation in the KIR loci and further evidence for the co-evolutin of KIR and HLA. Tissue Antigens 76, 9-17. doi: 10.1111/j.13990039.2010.01459.x

Hollenbach, J. A., Nocedal, I., Ladner, M. B., Single, R. M., and Trachtenberg, E. A. (2012). Killer cell immunoglobulin-like receptor (KIR) gene content variation in the HGDP-CEPH populations. Immunogenetics 64, 719-737. doi: 10.1007/s00251-012-0629-X

Hou, Y. F., Zhang, Y. C., Jiao, Y. L., Wang, L. C., Li, J. F., Pan, Z. L., et al. (2010). Disparate distribution of activating and inhibitory killer cell immunoglobulinlike receptor genes in patients with systemic lupus erythematosus. Lupus 19, 20-26. doi: 10.1177/0961203309345779

Ishiyama, K., Ohdan, H., Ohira, M., Mitsuta, H., Arihiro, K., and Asahara, T. (2006). Difference in cytotoxicity against hepatocellular carcinoma between liver and periphery natural killer cells in humans. Hepatology 43, 362-372. doi: 10.1002/hep. 21035

Karlsen, T. H., Boberg, K. M., Olsson, M., Sun, J. Y., Senitzer, D., Bergquist, A., et al. (2007). Particular genetic variants of ligands for natural killer cell receptors may contribute to the HLA associated risk of primary sclerosing cholangitis. J. Hepatol. 46, 899-906. doi: 10.1016/j.jhep.2007.01.032

Levinson, R. D., Okada, A. A., Ashouri, E., Keino, H., and Rajalingam, R. (2010). Killer cell immunoglobulin-like receptor gene-cluster 3DS1-2DL5-2DS1-2DS5 predisposes susceptibility to Vogt-Koyanagi-Harada syndrome in Japanese individuals. Hum. Immunol. 71, 192-194. doi: 10.1016/j.humimm.2009.11.001

Littera, R., Chessa, L., Onali, S., Figorilli, F., Secci, L., La Nasa, G., et al. (2016). Exploring the role of killer cell immunoglobulin-like receptors and their HLA class I ligands in autoimmune hepatitis. PLoS ONE 11:e0146086. doi: 10.1371/journal.pone.0146086 
Lucena, M. I., Molokhia, M., Shen, Y., Urban, T. J., Aithal, G. P., Andrade, R. J., et al. (2011). Susceptibility to amoxicillin-clavulanate-induced liver injury is influenced by multiple HLA class I and II alleles. Gastroenterology 141, 338-347. doi: 10.1053/j.gastro.2011.04.001

Moesta, A. K., Norman, P. J., Yawata, M., Yawata, N., Gleimer, M., and Parham, P. (2008). Synergistic polymorphism at two positions distal to the ligand-binding site makes KIR2DL2 a stronger receptor for HLA-C than KIR2DL3. J. Immunol. 180, 3969-3979. doi: 10.4049/jimmunol.180.6.3969

Moradi, S., Berry, R., Pymm, P., Hitchen, C., Beckham, S. A., Wilce, M. C., et al. (2015). The structure of the atypical killer cell immunoglobulin-like receptor, KIR2DL4. J. Biol. Chem. 290, 10460-10471. doi: 10.1074/jbc.M114.612291

Robinson, J., Waller, M. J., Stoehr, P., and Marsh, S. G. (2005). IPD-the immuno polymorphism database. Nucleic Acids Res. 33, D523-D526. doi: 10.1093/nar/gki032

Robles-Diaz, M., Lucena, M. I., Kaplowitz, N., Stephens, C., Medina-Caliz, I., González-Jimenez, A., et al. (2014). Use of Hy's law and a new composite algorithm to predict acute liver failure in patients with drug-induced liver injury. Gastroenterology 147, 109-118. doi: 10.1053/j.gastro.2014.03.050

Shaw, J., Hatano, H., and Kollnberger, S. (2014). The biochemistry and immunology of non-canonical forms of HLA-B27. Mol. Immunol. 57, 52-58. doi: 10.1016/j.molimm.2013.05.243

Stephens, C., López-Nevot, M. A., Ruiz-Cabello, F., Ulzurrun, E., Soriano, G., Romero-Gómez, M., et al. (2013). HLA alleles influence the clinical signature of amoxicillin-clavulanate hepatotoxicity. PLOS ONE 8:e68111. doi: 10.1371/journal.pone.0068111

Stern, M., Ruggeri, L., Capanni, M., Mancusi, A., and Velardi, A. (2008). Human leukocyte antigens A23, A24 and A32 but not A25 are ligands for KIR3DLI. Blood 112, 708-710. doi: 10.1182/blood-2008-02-137521

Stewart, C. A., Laugier-Anfossi, F., Vély, F., Saulquin, X., Riedmuller, J., Tisserant, A., et al. (2005). Recognition of peptide-MHC class I complexes by activating killer immunoglobulin-like receptors. Proc. Natl. Acad. Sci. U.S.A. 102, 13224-13229. doi: 10.1073/pnas.0503594102

Suppiah, V., Gaudieri, S., Armstrong, N. J., O’Connor, K. S., Berg, T., Weltman, M., et al. (2011). IL28B, HLA-C and KIR variants additively predict response to therapy in chronic hepatitis $\mathrm{C}$ virus infection in a European cohort: a cross-sectional study. PLoS Med. 8:e1001092. doi: 10.1371/journal.pmed. 1001092

Tian, Z., Chen, Y., and Gao, B. (2013). Natural killer cells in liver disease. Hepatology 57, 1654-1662. doi: 10.1002/hep.26115

Uhrberg, M. (2005). The KIR gene family: life in the fast lane of evolution. Eur. J. Immunol. 35, 10-15. doi: 10.1002/eji.200425743

Uhrberg, M., Valiante, N. M., Shum, B. P., Shilling, H. G., Lienert-Weidenbach, K., Corliss, B., et al. (1997). Human diversity in killer cell inhibitory receptor genes. Immunity 7, 753-763. doi: 10.1016/S1074-7613(00)80394-5

Winter, C. C., and Long, E. O. (1997). A single amino acid in the p58 killer cell inhibitory receptor controls the ability of natural killer cells to discriminate between the two groups of HLA-C allotypes. J. Immunol. 158, 4026-4028.

Conflict of Interest Statement: The authors declare that the research was conducted in the absence of any commercial or financial relationships that could be construed as a potential conflict of interest.

Copyright (c) 2016 Stephens, Moreno-Casares, López-Nevot, García-Cortés, MedinaCáliz, Hallal, Soriano, Roman, Ruiz-Cabello, Romero-Gomez, Lucena and Andrade. This is an open-access article distributed under the terms of the Creative Commons Attribution License (CC BY). The use, distribution or reproduction in other forums is permitted, provided the original author(s) or licensor are credited and that the original publication in this journal is cited, in accordance with accepted academic practice. No use, distribution or reproduction is permitted which does not comply with these terms. 